\title{
PRÁTICA HABITUAL DE ATIVIDADE FÍSICA E PREVALÊNCIA DE DOENÇAS CRÔNICO- DEGENERATIVAS EM ESTUDANTES DO CURSO DE EDUCAÇÃO FÍSICA
}

Felipe Henrique Mendes ${ }^{1}$, Giovani Ferreira da Silva ${ }^{1}$, Thiago Alves Garcia ${ }^{2}$, Guilherme Akio Tamura Ozaki ${ }^{2}$, Tatiana Emy Koike ${ }^{2}$, Aline Duarte Ferreira Ceccato ${ }^{1}$, Marcelo José Alves ${ }^{1}$, Éverton Alex Carvalho Zanuto ${ }^{1}$, Robson Chacon Castoldi ${ }^{1,3}$, Regina Celi Trindade Camargo ${ }^{4}$, José Carlos Silva Camargo Filho ${ }^{4}$

${ }^{1}$ Universidade do Oeste Paulista - UNOESTE, curso de Educação Física, Presidente Prudente, SP. ${ }^{2}$ Universidade Estadual Paulista - UNESP, Departamento de Educação Física, Presidente Prudente, SP. ${ }^{3}$ Universidade Estadual de Campinas - UNICAMP, Programa de Pós-Graduação em Ciências da Cirurgia, Campinas, SP. ${ }^{4}$ Universidade Estadual Paulista - UNESP, Departamento de Fisioterapia, Presidente Prudente, SP. E-mail: $\underline{\text { castoldi rc@yahoo.com.br }}$

\section{RESUMO}

O objetivo deste estudo foi analisar o nível de atividade física e a ocorrência de doenças crônicodegenerativas em estudantes do curso de Bacharelado em Educação Física. A amostra foi composta de 237 estudantes do curso de Educação Física. Foram aplicados o Questionário Internacional de Atividade Física (IPAQ) e um questionário de morbidade referida, baseado no "Standard Health Questionnaire for Washington State". Após a coleta dos dados, foram realizadas as análises de frequência e de associação pelo teste do Qui-Quadrado, com nível de significância de $5 \%$. Foi observado que dos avaliados $91,1 \%$ são ativos fisicamente e $8,9 \%$ são sedentários. Já para os casos de doenças crônico-degenerativas, foi verificado que os alunos se encontram com níveis de prevalência semelhantes aos observados a partir do histórico familiar. Nesse último caso, os achados demonstram que $27,8 \%$ são hipertensos, $24,5 \%$ sofrem de doenças metabólicas, 7,6\% sofrem de doenças cardiovasculares e $23,6 \%$ apresentam doenças osteomusculares.

Palavras-chave: atividade motora, exercício físico, estilo de vida sedentário; doença crônica.

\section{PHYSICAL ACTIVITY LEVEL AND CHRONICAL DEGENERATIVE DISEASE PREVALENCE IN STUDENTS OF PHYSICAL EDUCATION COURSE}

\begin{abstract}
The aim of this study was to identify the physical activity level and chronical degenerative disease prevalence in students of physical education course. The sample was consisted of 237 students of Physical Education. The International Physical Activity Questionnaire (IPAQ) and a morbidity questionnaire based on the Standard Health Questionnaire for Washington State were applied to carry out this study. After collecting the data, we performed the frequency and association analysis by Chi-square test, with significance level on $5 \%$. It was observed that $91.1 \%$ are physically active and $8.9 \%$ are sedentary; and the results of chronic diseases showed that students meet the same prevalence of illnesses that their parents, according to data on percentage. Also, the findings showed that $27.8 \%$ was hypertensive, $24.5 \%$ suffer of metabolic diseases, $7.6 \%$ and $23.6 \%$ suffer of cardiovascular and musculoskeletal diseases, respectively.
\end{abstract}

Keywords: motor activity, physical exercise, sedentary lifestyle, chronic disease.

\section{INTRODUÇÃO}

O aumento dos casos de doenças crônicas-degenerativas tem preocupado não apenas nações desenvolvidas, mas também países em desenvolvimento ${ }^{1}$. Um dos fatores desencadeadores dessas desordens é o sedentarismo. Além disso, a falta de atividade física está diretamente ligada ao surgimento de outras doenças. Uma pesquisa com norteamericanos mostrou que aproximadamente $12 \%$ dos casos de depressão, $18 \%$ dos casos de diabetes mellitus tipo II e $31 \%$ dos casos de 
doenças cardíacas, osteoporose e câncer de cólon, são atribuídos à inatividade física ${ }^{2}$.

Em contrapartida, a prática regular de atividade física é capaz de atuar de forma independente na prevenção de morbidades ${ }^{1,3}$. Estudos têm procurado destacar que hábitos da prática de atividade física incorporados na infância e adolescência podem transferir-se para as idades adultas ${ }^{4}$. Além disso, a prática desportiva pode ser um meio para diminuição do sedentarismo, uma vez que estimula o movimento e consequentemente $o$ gasto energético ${ }^{5}$

Cabe destacar que atividade física pode ser praticada em diferentes contextos do dia-adia. Embora seja realizada a partir de uma escolha pessoal e existência de condições favoráveis, prioritariamente está presente nos momentos de lazer, no trabalho, no ambiente doméstico ou como forma de deslocamento ${ }^{1 .}$.

Sabe-se que o exercício físico pode ser um aliado à melhoria da saúde e bem-estar do indivíduo ${ }^{6}$. Porém, estudos demonstram que a população brasileira apresenta elevados níveis de sedentarismo, podendo algumas regiões do país chegar a $70 \%$ da população ${ }^{7}$, abrange diferentes faixas etárias e de diferentes classes sociais ${ }^{1}$.

Segundo dados da Vigilância de Fatores de Risco e Proteção para Doenças Crônicas por inquérito telefônico (VIGITEL), a frequência de adultos que costumam despender três ou mais horas do dia vendo televisão variou entre $17,4 \%$ em Palmas-TO e 26,9\% em Macapá-AP. Entre homens, as maiores frequências foram encontradas em Macapá-AP (29,3\%), Belém-PA $(26,7 \%)$ e no Rio de Janeiro-RJ $(26,3 \%)$ e as menores em Palmas-TO $(17,6 \%)$, no Distrito Federal (18,6\%) e em Fortaleza-CE (18,7\%). Entre mulheres, as maiores frequências foram observadas em Salvador-BA $(26,4 \%)$, no Rio de Janeiro-RJ (25,7\%) e em Macapá-AP (24,8\%) e as menores em Curitiba-PR (15,9\%), Boa Vista-RR $(16,9 \%)$ e Palmas-TO $(17,2 \%)^{8}$.

Nesse sentido, observa-se que mesmo se tratando de uma população classificada como jovem, em que muitos casos não possuem responsabilidades familiares ou obrigações trabalhistas, se apresenta como sedentária". Outro ponto a ser levado em consideração é a realização de estudos em diversos cursos de graduação, não levando em conta a sua especificidade $^{10}$. Assim, faz-se necessária a realização de estudos voltados à área da saúde, em especial da Educação Física. Uma vez que está diretamente ligado à prescrição e incentivo à prática de atividades físicas.

Dessa forma, o objetivo do presente estudo foi analisar o nível de atividade física e ocorrências de doenças crônico-degenerativas em estudantes de um curso de bacharelado em Educação Física.

\section{METODOLOGIA}

Sujeitos

A amostra foi composta por estudantes do curso de bacharelado do 1 을 ao 8 o termos. A partir dos dados obtidos pela secretaria do curso de Educação Física, foi estabelecido o número de 540 alunos regulamente matriculados. A partir do cálculo amostral, foi definida a amostra de 250 alunos $\left(N_{\text {total }}=540\right)$ com base no número total de estudantes, estabelecendo-se um erro de $5 \%( \pm 5)$ e intervalo de confiança de $95 \%$ (IC=95\%). No entanto, 13 alunos não foram encontrados após três tentativas e foram assumidos como perda amostral, restando 237 avaliados.

Dessa forma, foi investigada uma amostra de no mínimo 30 alunos de cada uma das salas. Após terem aceitado responder o questionário, foram distribuídos para os alunos, o Termo de Consentimento Livre e Esclarecido (TCLE). Este estudo está de acordo com a resolução 466/2012 da CONEP e foi submetido à aprovação pelo Comitê de Ética em Pesquisa da Universidade do Oeste Paulista - UNOESTE, tendo recebido o protocolo de aprovação de núm. 2437.

Instrumentos de avaliação

Prática habitual de atividade física

Foi avaliada pelo Questionário Internacional de Atividade Física (IPAQ). Este instrumento detém-se de validade internacional e possui duas versões, curta e estendida. No presente estudo foi utilizada a versão curta do IPAQ (Short Version), utilizado previamente por Pardini et al. ${ }^{11}$ e Benedetti et al. ${ }^{12}$.

Foram analisados os níveis de atividade física entre sexo masculino e sexo feminino, e comparado entre os diferentes termos, para se identificar as diferenças entre eles. $O$ ponto de corte para o indivíduo ser classificado como fisicamente ativo foi de no mínimo 150 minutos semanais, com a realização de atividade física em pelo menos cinco dias da semana, englobados no tempo livre (lazer), trabalho ou transporte. 
Morbidade referida

A ocorrência de doenças crônicodegenerativas foi investigada a partir de um questionário de morbidade referida baseado no "Standard Health Questionnaire for Washington State" (WSHIP) ${ }^{13}$. Assim, foi possível verificar a prevalência de doenças crônicas no indivíduo avaliado e em seus familiares (pai e mãe). 0 questionário investiga a ocorrência de doenças em um período de até 10 anos.

Análise estatística

Após a obtenção dos dados, os avaliados foram classificados entre ativos e sedentários e observada a ocorrência de doenças crônicas. A análise de frequência foi estabelecida a partir de cada um dos termos avaliados, estabelecendo-se as frequências absoluta e relativa (\%). Foi realizada a análise de associação pelo teste do Qui-Quadrado para se verificar a associação entre as variáveis "prática de atividade física" com "ocorrência de doenças" nos estudantes e familiares (pais). Para isso foi admitido um erro de $5 \%$. Foi utilizado o Software com pacote estatístico SPSS 17.0.

\section{RESULTADOS}

Para obtenção dos resultados foram avaliados 237 alunos, sendo 168 do sexo masculino e 69 do sexo feminino. Os resultados abaixo mostram as frequências absoluta e relativa de avaliados por termo (Tabela 1 ).

Tabela 1. Distribuição de participantes nos diferentes termos.

\begin{tabular}{ccc}
\hline Termos & Frequência & Válidos (\%) \\
\hline 1 & 30 & 12,7 \\
2 & 29 & 12,3 \\
3 & 30 & 12,7 \\
4 & 30 & 12,7 \\
5 & 31 & 13,1 \\
6 & 26 & 11,0 \\
7 & 30 & 12,7 \\
8 & 30 & 12,7 \\
Total & 237 & 100 \\
\hline
\end{tabular}

Quando observados a partir do sexo, foi verificado maioria de indivíduos do sexo masculino, com prevalência com $71 \%$ dos avaliados, enquanto o sexo feminino representou $29 \%$ dos avaliados. Os estudantes foram classificados em sedentários e ativos, de acordo com o protocolo utilizado, foi encontrado que $91,1 \%$ dos avaliados foram considerados ativos e $8,9 \%$ considerados sedentários (Tabela 2 ).

Tabela 2. Distribuição de participantes por sexo e estilo de vida.

\begin{tabular}{lccc|ccc}
\hline & \multicolumn{3}{c|}{ Sexo } & \multicolumn{3}{c}{ Estilo de vida } \\
\hline Frequência & Masculino & Feminino & Total & Sedentário & Fisicamente Ativo & Total \\
Freq \% & 168 & 69 & 237 & 21 & 216 & 237 \\
\hline
\end{tabular}

Após a análise a partir da intensidade das atividades, foi verificado que a atividade leve, praticada por cinco dias na semana, foi relatada com maior frequência. Foi verificado que $45,9 \%$ dos entrevistados fazem atividade moderada com frequência igual a cinco vezes na semana e que $38,4 \%$ praticam atividade vigorosa com frequência igual a cinco vezes na semana (Tabela $3)$. 
Tabela 3. Frequência da prática de atividades leves, moderadas e vigorosas.

\begin{tabular}{cccc}
\hline Atividade & Leve & Moderada & Vigorosa \\
\hline Dias/semana & Frequência (\%) & Frequência (\%) & Frequência (\%) \\
\hline 0 & $25(10,5)$ & $31(13,1)$ & $43(18,1)$ \\
1 & $8(3,4)$ & $11(4,6)$ & $27(11,4)$ \\
2 & $22(9,3)$ & $34(14,3)$ & $32(13,5)$ \\
3 & $24(10,1)$ & $36(15,2)$ & $29(12,2)$ \\
4 & $15(6,3)$ & $16(6,8)$ & $15(6,3)$ \\
5 & $82(34,6)$ & $56(23,6)$ & $53(22,4)$ \\
6 & $23(9,7)$ & $25(10,5)$ & $29(12,2)$ \\
7 & $38(16,0)$ & $27(11,4)$ & $9(3,8)$ \\
Total & $237(100)$ & $237(100)$ & $237(100)$ \\
\hline
\end{tabular}

Sobre a ocorrência de hipertensão arterial nos estudantes e em seus familiares foi observado que $27,8 \%$ possuem a doença, enquanto $26,2 \%$ dos pais são hipertensos (Tabela 4).

Tabela 4. Prevalência de hipertensão arterial nos participantes e nos pais.

\begin{tabular}{lcc}
\hline & Participantes & Pai ou mãe \\
\hline & Frequência (\%) & Frequência (\%) \\
\hline Ausência & $171(72,2)$ & $175(73,8)$ \\
Presença & $66(27,8)$ & $62(26,2)$ \\
Total & $237(100)$ & $237(100)$ \\
$\mathbf{X}^{\mathbf{2}}$ & 0,6 & 0,4 \\
\hline
\end{tabular}

Sobre as doenças metabólicas, $24,5 \%$ dos estudantes avaliados sofrem de alguma delas, enquanto $26,2 \%$ dos pais também apresentam estas doenças (Tabela 5).

Tabela 5. Prevalência de doenças metabólicas nos participantes e nos pais.

\begin{tabular}{lcc}
\hline & Participantes & Pai ou mãe \\
\hline & Frequência (\%) & Frequência (\%) \\
\hline Ausência & $179(75,5)$ & $178(75,1)$ \\
Presença & $58(24,5)$ & $59(24,9)$ \\
Total & $237(100)$ & $237(100)$ \\
$\mathbf{X}^{\mathbf{2}}$ & 0,3 & 0,7 \\
\hline
\end{tabular}

Foi observado que $7,6 \%$ dos estudantes avaliados se encontram com doenças cardiovasculares. Além disso, $7,2 \%$ dos pais apresentam estas doenças (Tabela 6).

Tabela 6. Prevalência de doenças cardiovasculares nos participantes e nos pais.

\begin{tabular}{lcc}
\hline & Participantes & Pai ou mãe \\
\hline & Frequência (\%) & Frequência (\%) \\
\hline Ausência & $219(92,4)$ & $220(92,8)$ \\
Presença & $18(7,6)$ & $17(7,2)$ \\
Total & $237(100)$ & $237(100)$ \\
$\mathbf{X}^{2}$ & 0,2 & 0,4 \\
\hline
\end{tabular}

Foi verificado também que $23,6 \%$ dos alunos avaliados se encontram com doenças osteomusculares. Já nos pais, a ocorrência foi de 21,5\% (Tabela 7). 
Tabela 7. Prevalência de doenças osteomusculares nos participantes e nos pais.

\begin{tabular}{lcc}
\hline & Participantes & Pai ou mãe \\
\hline & Frequência $(\%)$ & Frequência $(\%)$ \\
\hline Ausência & $181(76,4)$ & $186(78,5)$ \\
Presença & $56(23,6)$ & $51(21,5)$ \\
Total & $237(100)$ & $237(100)$ \\
$\mathbf{X}^{2}$ & 0,005 & 0,03 \\
\hline
\end{tabular}

\section{DISCUSSÃO}

Objetivo do estudo foi analisar o nível de atividade física e ocorrências de doenças crônico degenerativas em estudantes de um curso de bacharelado em Educação física. Foi observado que $8,9 \%$ dos indivíduos foram classificados como sedentários ou insuficientemente ativos e 91,1\% definidos como fisicamente ativos.

O presente estudo utilizou o Questionário Internacional de Atividade física (IPAQ) como forma de obtenção dos resultados. Tal instrumento possui validação internacional e dessa forma, permite a comparação entre diferentes regiões ou países ${ }^{11,12}$.

Foi verificado que pequena parcela dos avaliados foi classificada como sedentários ou insuficientemente ativos. Tal fato pode ser explicado pela amostra ser composta por estudantes de educação física e, além disso, serem em sua maioria adultos jovens, fato que pode indicar fator de proteção contra surgimento de possíveis doenças ${ }^{7}$.

No entanto, tais resultados não retratam a realidade da população brasileira, uma vez que determinadas regiões do Brasil podem apresentar até $70 \%$ de indivíduos inativos fisicamente ${ }^{1,14}$. Isso faz com que haja a preocupação das autoridades e órgãos governamentais com relação a inatividade física.

A pesquisa realizada por Rodrigues et al. ${ }^{15}$ em estudantes universitários, verificou que aproximadamente $30 \%$ dos avaliados eram sedentários. Apesar dos autores considerarem este percentual baixo, representa um terço dos entrevistados.

Outras investigações que se propuseram a analisar estudantes universitários verificaram prevalência de inatividade física em $49,1 \%$ dos indivíduos do sexo masculino e $77,9 \%$ nos entrevistados do sexo feminino ${ }^{16}$. Percebe-se que, além de elevados valores percentuais de indivíduos inativos no horário de lazer, em alguns casos, o gênero pode ser determinante na prática habitual de atividade física ${ }^{9}$.
Já o estudo de Fontes e Vianna ${ }^{7}$, conduzido em uma universidade pública, verificou que a prevalência de baixo nível de atividade física foi de $31,2 \%$ em universitários. Além disso, os estudantes do período noturno e aqueles que passam menos tempo na universidade obtiveram maior prevalência de baixo nível de atividade física. Neste último caso, os autores destacam o trabalho e rotina diária como principais fatores para a falta de prática de exercícios físicos.

O presente estudo também analisou a prevalência de doenças nos estudantes e seus familiares (pai e mãe). Foi verificada a prevalência de doenças nos participantes e pais, respectivamente, de $27,8 \%$ e $26,2 \%$ para hipertensão arterial sistêmica, de $24,5 \%$ e $24,9 \%$ para doenças metabólicas, de 7,6\% e 7,2\% para doenças cardiovasculares e de $23,6 \%$ e $21,5 \%$ para doenças Osteomusculares.

A partir desses achados, é possível verificar que aproximadamente um quarto (25\%) dos entrevistados possuíam algum tipo de doença. Outro fato observado foi a estreita relação com o histórico familiar, no qual valores semelhantes foram observados entre os avaliados e os pais.

Nesse sentido, verifica-se que apesar de jovens, um quarto dos entrevistados possui algum tipo de doença e tal fato se assemelha aos familiares. Esses achados apontam para a necessidade da prevenção no surgimento de doenças ainda na infância e adolescência, uma vez que é possível observar relação direta entre hábitos praticados na infância e adolescência permanecentes até a idade adulta ${ }^{17-19}$.

Outro ponto a ser destacado é o exercício físico como forma de combate às doenças crônicas. Sabe-se que a atividade física atenua os sintomas de diversas doenças como hipertensão arterial sistêmica, diabetes melitus tipo II, osteoporose e obesidade ${ }^{20,21}$. Nesse sentido, a prática de exercícios físicos deve ser incentivada nessa população com a finalidade de prevenir ou combater agravos degenerativos, uma vez que, 
por possuir característica crônica, tais doenças tendem a aparecer com o avançar da idade. Tais medidas além de gerar benefícios à saúde podem diminuir gastos com o sistema de saúde pública, uma vez que diminui as chances de incidências de doenças.

Sendo assim, o presente estudo contribui na identificação do nível de atividade física de estudantes universitários e a prevalência de doenças crônico degenerativas em estudantes e familiares. No entanto, algumas limitações devem ser consideradas, como a área de formação, nesse caso, a educação física e o grau de parentesco investigado. Estudos futuros que busquem investigar estudantes de outras áreas do conhecimento podem vir a colaborar com o presente estudo.

Dessa maneira, conclui-se que os avaliados se apresentaram, em sua maioria, fisicamente ativos. Além disso, a amostra analisada apresenta relação entre os casos de doenças crônico-degenerativas com histórico familiar de doenças.

\section{CONFLITO DE INTERESSE}

Os autores declaram não haver qualquer potencial conflito de interesse que possa interferir na imparcialidade deste trabalho científico.

\section{REFERÊNCIAS}

1. Del Duca GF, Rombaldi AJ, Knuth AG, Azevedo MR, NAhas MV, Hallal PC et al. Associação entre nível econômico e inatividade física em diferentes domínios. Rev Bras Ativ Fís e Saúde. 2009; 14(2):12331. DOI:

http://dx.doi.org/10.12820/rbafs.v.14n2p123-131

2. Hallal, PC, Dumith SC, Bastos JP, Reichert FF, Siqueira FV, Azevedo MR et al. Evolução da pesquisa epidemiológica em atividade física no Brasil: revisão sistemática. Rev Saúde Pública. 2007;41(3):453-60. DOI: $\quad$ http://dx.doi.org/10.1590/5003489102007000300018

3. Ho SS, Dhaliwal SS, Hills AP, Pal S. The effect of 12 weeks of aerobic, resistance or combination exercise training on cardiovascular risk factors in the overweight and obese in a randomized trial. BMC Public Health. 2012;12:704. DOI: http://dx.doi.org/10.1186/1471-2458-12-704

4. Chu CK, Wong MS. Comparison of prosthetic outcomes between adolescent transtibial and transfemoral amputees after Sichuan earthquake using Step Activity Monitor and Prosthesis Evaluation Questionnaire. Prosthet Orthot Int. 2016;40(1):58-64. DOI: http://dx.doi.org/10.1177/0309364614556837

Colloq Vitae2017 set-dez; 9(3): 59-65. DOI: 10.5747/cv.2017.v09.n3.v210 ISSN 1984-6436/C 2017 - Publicado pela Universidade do Oeste Paulista.

Artigo Open Access sob uma licença CC BY-NC-ND (http://creativecommons.org/licenses/by-nc-nd/4.0/).
5. Shiraev T, Barclay G. Evidence based exercise clinical benefits of high intensity interval training. Aust Fam Physician. 2012;41(12):960-2.

6. Cieslak F, Levandoski G, Gôes SM, Santos TK, Junior GBV, Leite N. Relação do nível de qualidade de vida e atividade física em acadêmicos de Educação Física. Fit Perf J. 2007;6(6):357-61. DOI: http://dx.doi.org/10.3900/fpj.6.6.357.p

7. Fontes ACD, Vianna RPT. Prevalência e fatores associados ao baixo nível de atividade física entre estudantes universitários de uma universidade pública da região Nordeste-Brasil. Rev Bras Epidemiol. 2009;12(1):20-9. DOI: http://dx.doi.org/10.1590/S1415790X2009000100003

8. Vigilância de fatores de risco e proteção para Doenças Crônicas por inquérito telefônico - VIGITEL. Secretaria de Vigilância em Saúde, 2015.

9. Mielke GI, Ramis TR, Habeyche EC, Oliz MM, Tessmer MGS, Azevedo MR et al. Atividade física e fatores associados em universitários do primeiro ano da Universidade Federal de Pelotas. Rev Bras Ativ Fís e Saúde. 2010;15(1):57-64.

10. Silva DAS. Nível de atividade física e fatores associados em acadêmicos de Educação Física de uma Universidade Pública do nordeste do Brasil. Rev Bras Ativ Fís e Saúde. 2011;16(3):193-8.

11. Pardini R, Matsudo SMM, Araújo T, Matsudo V, Andrade $\mathrm{E}$, Braggion $\mathrm{G}$ et al. Validação do questionário internacional de nível de atividade física (ipaq - versão 6): estudo piloto em adultos jovens brasileiros. Rev Bras Cien Mov. 2001;9(3):45-51. DOI: http://dx.doi.org/10.18511/rbcm.v9i3.393

12. Benedetti TRB, Antunes PC, Añez CRR, Mazo GZ, Petroski EL. Reprodutibilidade do questionário internacional de atividade física (IPAQ) em homens idosos. Rev Bras Med Esporte. 2007;13(1):11. DOI: http://dx.doi.org/10.1590/S1517$\underline{86922007000100004}$

13. Washington State Health Insurance Pool. Standard Health Questionnaire for Washington State, WSHIP, Washington, 2008.

14. Guedes DP, Gonçalves LAVV. Impacto da prática habitual de atividade física no perfil lipídico de adultos. Arq Bras Endo e Meta. 2007;51(1):72-8. DOI: http://dx.doi.org/10.1590/S000427302007000100012

15 Rodrigues ESR, Cheik NC, Mayer, AF. Nível de atividade física e tabagismo em universitários. Rev Saúde Pública. 2008;42(4):672-8. DOI: http://dx.doi.org/10.1590/S003489102008000400013 
16. Pitanga FJG, Lessa I. Prevalência e fatores associados ao sedentarismo no lazer em adultos. Cad Saúde Pública. 2005;21(3):870-7. DOI: http://dx.doi.org/10.1590/S0102311X2005000300021

17. Silva GSF, Bergamaschine $R$, Rosa $M$, Melo $C$, Miranda R, Filho MB. Avaliação do nível de atividade física de estudantes de graduação das áreas saúde/biológica. Rev Bras Med Esporte. 2007;13(1):39-42. DOI:

http://dx.doi.org/10.1590/S1517-

86922007000100009

18. Souza TF, Nahas MV. Prevalência e fatores associados a menores níveis de prática de atividades físicas no lazer em estudantes de uma universidade pública do Estado da Bahia. Rev Bras Ativ Fís e Saúde. 2012;16(4):322-9.

19. Brito WF, Santos CL, Marcolongo AA, Campos MD, Bocalini DS, Antonio EL, et al. Nível de atividade física em professores da rede estadual de ensino. Rev Saúde Pública. 2012;46(1):104-9. DOI: http://dx.doi.org/10.1590/S0034$\underline{89102012000100013}$

20 Trapp EG, Chisholm DJ, Freund J, Boutcher SH. The effects of high-intensity intermittent exercise training on fat loss and fasting insulin levels of young women. Int J Obesity. 2008;32:684-91. DOI: http://dx.doi.org/10.1038/sj.ijo.0803781

21. Pereira $M$, Lunet $N$, Azevedo A, Barros $H$. Differences in Prevalence, awareness, treatment and control of hypertension between developing and developed countries. J Hipertens. 2009;27(5):963-75.

Recebido para publicação em 04/11/2016

Revisado em 26/04/2017

Aceito em 22/03/2018 